\title{
A procedure to quantify hypothalamically elicited agonistic behavior in the cat'
}

CORNELIS L. J. STOKMAN AND MURRAY GLUSMAN, NEW YORK STATE PSYCHIATRIC INSTITUTE, 722 West 168th Street, New York 10032

A procedure is described which permits quantification of hypothalamically elicited flight and attack responses. The procedure is sensitive to stimulus parameters and to the effects of lesions in brain structures. Data are presented illustrating its use in studying facilitation of hypothalamic flight by simultaneous amygdaloid stimulation.

Electrical stimulation of certain hypothalamic areas produces responses which can be categorized as agonistic behavior, i.e., behavior displayed by an organism in response to a threatening stimulus, e.g., attack and flight reactions. Description of these responses and localization of the anatomical regions from which they could be obtained were the main concern of many of the earlier investigators (Hess, 1957; Hunsperger, 1956; Glusman \& Roizin, 1960).

However, in most of these studies no attempt was made to obtain a quantitative measurement of the responses or to specify the functional relationships between stimulus parameters and response strength. We have been studying hypothalamically elicited agonistic behavior and its functional properties and have developed in our laboratory a behavioral procedure which permits a quantitative approach to these problems. It is, therefore, possible to assess the effects of both neural and environmental variables on the strength of these responses. The procedure is applicable to both attack and flight responses and provides a means of studying the interaction between various brain structures in the regulation of hypothalamically elicited agonistic responses. An illustration of such an application is presented below.

The example focuses on amygdaloid regulation of hypothalamic flight, but the use of the procedure in investigating hypothalamic attack is also demonstrated. Essentially, response latencies of hypothalamic flight or attack are determined and changes in these latencies by simultaneous stimulation of various amygdaloid loci observed.

Method. The experimental chamber consists of a rectangular aluminum box ( 34 in. $x 30$ in. $x 22$ in.) divided into two identical compartments separated by a removable barrier of variable height (Fig. 1). A small transparent rat cage is fitted into each end wall. It is so constructed that light pressure against the movable front panel activates a microswitch which interrupts stimulating and timing circuits.

A cat with appropriately implanted bipolar electrodes in the hypothalamus and other sites is placed in the chamber and attached via a light multiple lead cable and stimulus isolation units to the outputs of two Grass square-wave stimulators. The output of one stimulator goes to the hypothalamic site and the output of the second to a test site, e.g., the amygdala. For stimulation, biphasic square-wave pulses at a frequency of $60 \mathrm{cps}$ and pulse duration of $0.75 \mathrm{msec}$ are employed. To guard against the complex stimulus effects of introducing two electrical pulses into the brain at the same time, the outputs of the stimulators are interdigitated so that during "simultaneous" stimulation a pulse to one site is alternated with a pulse to the second site in rapid succession, with a delay of 8.5 msec. Stimuli are routinely monitored via an oscilloscope and current probes for voltage and current.

Utilizing this arrangement a stimulus intensity is selected of sufficient strength to elicit either attack or flight from the hypothalamic site at a latency falling between 5-10 sec. Stimulus intensities for the second site are chosen at subthreshold levels, which will not elicit any observable behavioral effects or electrical after-discharges. EEG monitoring was routinely performed to observe possible after-discharges.

Attack Responses. To study regulation of attack responses a rat is placed in the rat cage, and the cat in the testing chamber is stimulated via the hypothalamic electrodes alone. The onset of the stimulation automatically activates a Sodeco print-out counter. With a properly implanted animal (i.e., electrodes in the vicinity of the ventromedial nucleus of the hypothalamus) and a properly selected stimulus intensity, the cat after a certain latency will attack the rat. In doing so it strikes the movable panel of the rat cage and closes the microswitch, thereby terminating the stimulus and stopping the timer and the print-out counter. The latency for the response to hypothalamic stimulation alone is thus recorded. In the next trial the hyputhalamus and the second site, e.g., amygdala, are stimulated simultaneously and the latency for attack is again determined. In a given session 20 trials are presented, alternating in $A B A B$ fashion, with an intertrial interval of $3 \mathrm{~min}$.

Flight Responses. To study flight responses the same test clamber is used, but this time a barrier, $10 \mathrm{in}$. high, is placed in the center of the chamber, partially dividing it into two identical compartments. In suitably implanted cats hypothalamic stimulation will elicit flight and not attack. For investigative purposes flight is operationally defined as the $S$ leaping the barrier in response to hypothalamic stimulation. The chamber is so arranged that in keaping the barrier the animal breaks a light beam, which via a photoelectric relay terminates the stimulus and stops the timer and print-out counter. Latencies for flight following hypothalamic stimulation are thus recorded. When sludying amygda loid regulation of hypothalamic flight, stimulation of the hyputhalamus alone is alternated with simultaneous hypothalamic and amygdaloid slimulation.

To make certain that the effect of the simultaneous amygdaloid stimulation on hypothalamic flight or attack is truly a neural phenomenon and not the result of some complex electrical artifacts, the amygdaloid sites are destroyed after completion of the stimulation sessions by means of anodal electrolytic lesions created via the implanted amygdaloid electrodes. The $S$ s are permitted to recover from the effects of the lesions and are then re-tesled using the same procedure employed in the pre-lesion sessions. Upon completion of the investigation the animals are sacrificed and the brains are prepared for microscopic histological examination so that the stimulation sites may be identified precisely and the extent of the amygdaloid lesions delermined.

Results and Discussion. The data presented illustrate the use of this procedure to study the effects of amygdaloid stimulation on hypothalamically elicited flight and attack responses. Figure $2 \mathrm{~A}$ shows the latencies for flight elicited by hypothalamic stimulation alone contrasted with latencies following simultaneous amygdaloid and hypothalamic stimulation. Sub-threshold amygdaloid stimulation markedly facilitates hypothalamic flight, as can be seen in the shorter latencies following the simultaneous stimulation. In addition to differences in response latencies following hypothalamic and combined hypothalamic and amygdaloid stimulation this figure reveals another interesting phenomenon. Typically, the hypothalamic response latencies tend to increase with repeated stimulus presentation. Although systematic investigation of this habituation effect was not attempted, we have noted that the degree of

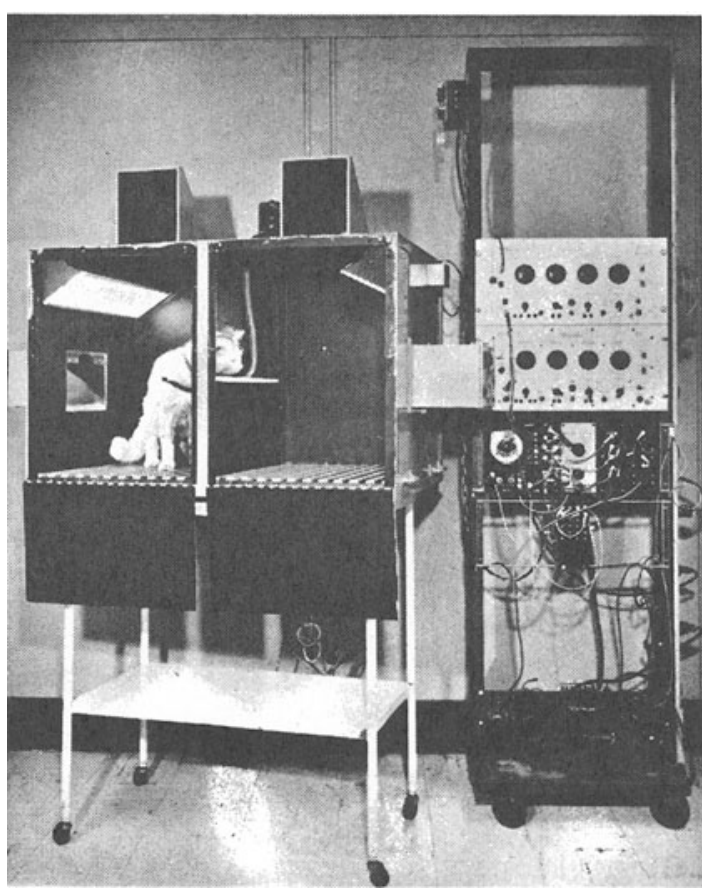

Fig. 1. Photograph of testing chamber with upper part of front panel removed. 

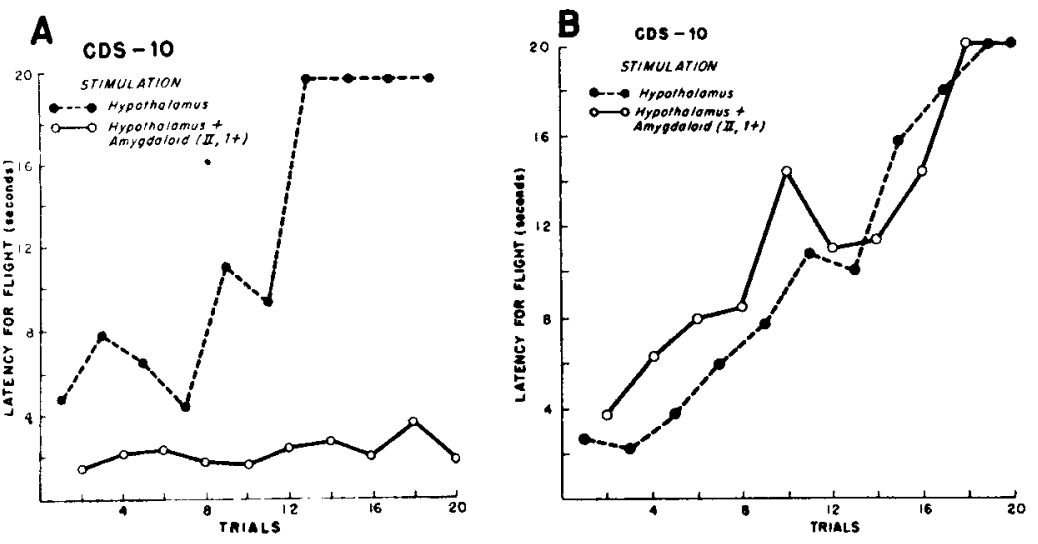

Fig. 2. Latencies for flight following hypothalamic and simultancous hypothalamic and amygdaloid stimulation before (2A) and after (2B) destruction of amygdaloid site; attack latencies following hypothalamic and simultaneous hypothalamic and amygdaloid stimulation (2C); diagram indicating placement of amygdaloid electrodes (2D). Key to abbreviations: Abm: N. amygdaloideus basalis (pars magnocellularis); Abp: N. amygdaloideus basalis (pars parvocellularis); ACL: N. amygdaloideus centralis (pars lateralis); AL: $\mathbf{N}$. amygdaloideus lateralis; $C E$ : capsula externa; CI: capsula interna; CL: claustrum; EN: N. entopeduncularis; F. Rhin: fissura rhinalis; Hip: hippocampus; Pir: lobus piriformis; Put: Putamen; TO: tractus opticus.

habituation seems inversely related to the stimulus intensity and the intertrial interval.

Figure $2 \mathrm{~B}$ presents data for the same animal after the amygdaloid site was destroyed by creating a lesion at the tip of the amygdaloid electrodes. Destruction of the amygdaloid site completely abolished the facilitation, demonstrating that the facilitation depended on the integrity of the amygdaloid site and that it could not be attributed to electrical artifacts. The placement of the amygdaloid electrodes is shown in Fig. 2D. The facilitating site (black circle) was in the lateral nucleus, whereas a control placement (black triangle) in the pyriform cortex did not change hypothalamic attack or flight latencies. The hypothalamic electrodes of this animal were located in the anterior hypothalamus (flight) and in the ventromedial nucleus (attack).

The flight-facilitating amygdaloid site had no effect on hypothalamic attack as illustrated in Fig. 2C. It appears, therefore, that amygdaloid loci which influence flight do not necessarily coincide with those influencing attack responses.

Functional relationships between amygdaloid and hypothalamic structures have been demonstrated electrophysiologically (Dreifuss et al, 1967; Gloor, 1955; Sawa et al, 1958), but with the exception of the work by Flynn and coworkers, neural regulation of hypothalamic agonistic responses, has not been demonstrated at the behavioral level. Flynn and coworkers confined their studies to hypothalamic attack and reported that these responses could be facilitated or suppressed by simultaneous stimulation of various amygdaloid, thalamic, hippocampal, and mesencephalic loci (Egger \& Flynn, 1903; MacDonnel \& Flynn, 1964; Siegel \& Flynn, 1968; Sheard \& Flynn, 1967).

The procedure presented here provides not only a refined approach to the study of such responses, but makes it possible for the first time to investigate the regulation of hypothalamically elicited flight responses in a systematic and quantitative fashion. It, therefore, enables investigation of brain elicited, complex emotional responses to move forward from the problems of description and localization of responses, which occupied the pioneering investigators in this area almost exclusively, to questions of functional relationships and regulatory phenomena.

Because we are dealing with highly complex phenomena, full understanding of the nature of hypothalamic flight and attack responses requires an understanding of the possible aversive aspects of both hypothalamic and amygdaloid stimulation. It is conceivable that hypothalamic flight is due to the aversive properties of the stimulation and that these aversive properties are enhanced by simultaneous amygdaloid stimulation. Determination of the possible aversiveness of the stimulation is therefore essential before an interpretation of these responses in terms of instrumental escape responses or in terms of unconditioned, elicited reactions can be made. Current research in our laboratory suggests that hypothalamic flight responses are not under the control of the negatively reinforcing properties of the stimulation, but instead they should be viewed as reactions, controlled primarily by the eliciting properties of the stimulation.

\section{REFERENCES}

DREIFUSS, J. J., MURPHY, J. T., \& GLOOR, P. Constrasting effects of two identified amygdaloid efferent pathways on single hypothalamic neurons. $J$. Neurophysiol, 1968, 31, 237-248.

EGGER, M. D., \& FLYNN, J. P. Effects of electrical stimulation of the amygdala on hypothalamically elicited attack behavior in cats. $J$. Neurophysiol., 1963,26, 705-720.

GLOOR, P. Electrophysiological studies on the connections of the amygdaloid nucleus in the cat. Part 2: The electrophysiological properties of the amygdaloid projection system. EEG clin. Neurophysiol, 1955, 7, 243-264.

GLUSMAN, M., \& ROIZIN, L. Role of the hypothalamus in the organization of agonistic behavior in the cat. Trans. Amer. Neurol. Assoc., 1960, 85, 177-181.

HESS, W. R. The functional organization of the diecephalon. New York: Grune \& Stratton, 1957.

HUNSPERGER, R. W. Affektreaktionen auf elektrische Reizung im Hirnstamm der Katze. Helvetica physiologica et pharmacologica acta, 1956, 14, 70-92.

MacDONNEL, M. F., \& FLYNN, J. P. Attack elicited by stimulation of the thalamus in cats. Science, $1964,44,1249-1250$.

SAWA, M., MARUYAMA, N., HANAI, T., \& KAJI, S. Regulatory influence of amygdaloid nuclei upon the unitary activity in ventromedial nucleus of hypothalamus. Folia psychiat. Neurol Jap. 1959, 13, 235-256.

SHEARD, M. H., \& FLYNN, J. P. Facilitation of attack behavior by stimulation of the midbrain of cats. Brain Res, 1967,4,324-333.

SIEGEL, A., \& FLYNN, J. P. Differential effects of electrical stimulation and lesions of the hippocampus and adjacent regions upon attack behavior in cats. Brain Res., 1968, 7, 252-267.

\section{NOTE}

1. This research was supported by Grant MH-03660 from the National Institute of Mental Health. 